\title{
SEMI-IDEMPOTENT MEASURES ON ABELIAN GROUPS ${ }^{1}$
}

\author{
BY IRVING KESSLER
}

Communicated by E. Hewitt, September 26, 1966

Let $M(G)$ denote the set of complex valued regular Borel measures on a compact abelian group $G$. We assume that $\Gamma$, the dual group of $G$, is a totally ordered group. Let $F(G)$ denote the set of all $\mu \in M(G)$ such that the Fourier transform $\hat{\mu}$ of $\mu$ is an integer valued function. A measure $\mu$ is idempotent if $\hat{\mu}$ assumes only the values 0 or 1 . A $\mu \in M(G)$ is semi-idempotent if $\hat{\mu}(\gamma)=0$ or 1 for all $\gamma>0$ in $\Gamma$.

The purpose of this note is to sketch a proof of the following theorem.

THEOREM 1. If $\mu \in M(G)$ and $\hat{\mu}(\gamma)$ is an integer for all $\gamma>0$ in $\Gamma$, then there exists a $\lambda \in F(G)$ such that $\lambda(\gamma)=\hat{\mu}(\gamma)$ for all $\gamma>0$. In particular, if $\mu$ is a semi-idempotent measure on $G$, then there exists an idempotent measure $\lambda$ on $G$ such that $\hat{\mu}(\gamma)=\hat{\lambda}(\gamma)$ for all $\gamma>0$ in $\Gamma$.

This result was obtained by Helson [3], for the case $G=T$, the circle group, $\Gamma=Z$ the integer group. Also a special case of Theorem 1 for $G=T^{2}, \Gamma=Z^{2}$ was proven by Rudin [6].

OutLine of Proof. We assume first that $G=T^{k}$, the $k$-dimensional torus group and $\Gamma=Z^{k}$ is a totally ordered group. Then $Z^{k}=\Gamma_{1} \oplus \Gamma_{2}$ where $\Gamma_{2}$ is a subgroup of the reals, $\Gamma_{1}$ is a totally ordered group, and $\Gamma_{1} \oplus \Gamma_{2}$ is lexicographically ordered from the right. Let $\mu \in M(G)$ such that $\hat{\mu}(\gamma)$ is an integer for all $\gamma>0$ in $Z^{k}$.

Let $E(\mu)=\left\{\gamma \in Z^{k}: \gamma>0\right.$ and $\left.\hat{\mu}(\gamma) \neq 0\right\}$ and, for every positive integer $n$, let

$$
A_{n}=\left\{\gamma \cdot \mu \in M(G): \gamma=\left(\gamma_{1}, \gamma_{2}\right) \in E(\mu) \text { and } \gamma_{2}>n\right\} .
$$

We then prove the following

Lemma. If $E(\mu)$ and $A_{n}$ are defined as above, we have either

(1) $E(\mu)$ is contained in a finite union of $(k-1)$-dimensional hyperplanes, or

(2) $A_{n} \neq \varnothing$ for every $n$.

If (1), then Theorem 1 follows by induction. If (2), we set

1 This is an announcement of a portion of the author's dissertation at the University of Wisconsin written under the direction of Professor Walter Rudin. 


$$
A=\bigcap_{n=1}^{\infty} \bar{A}_{n}
$$

$\left(\bar{A}_{n}\right.$ is the weak*-closure of $\left.A_{n}\right)$. Then $A$ is nonempty and weak*compact and hence contains an element $\nu \neq 0$, of minimal norm. Then $\nu \in F(G)$, and applying a lemma of Ito and Amemiya [5], we find that $\nu$ is a measure of the form $\gamma \cdot \chi_{H} \mu$ where $\chi_{H}$ is the characteristic function of a compact subgroup $H$ of $G$, and $\gamma \in \Gamma$.

Let $\mu_{1}=\chi_{H} \mu$. Then $\mu=\mu_{1}+\left(\mu-\mu_{1}\right)$ is an orthogonal decomposition of $\mu$ where $\mu_{1} \in F(G)$ and $\left(\hat{\mu}-\hat{\mu}_{1}\right)(\gamma)$ is an integer for all $\gamma>0$ in $\Gamma$. Now we can apply the argument above to the measure $\mu-\mu_{1}$. Since the norm of $\mu-\mu_{1}$ decreases at least one from that of $\mu$, we see that after a finite number of steps, we obtain

$$
\mu=\sum_{i=1}^{n} \mu_{i}+\tau
$$

where $\mu_{i} \in F(G)$ for $i=1, \cdots, n$, and $\hat{\tau}(\gamma)=0$ for all $\gamma>0$.

The proof of the theorem for arbitrary compact $G$ is completed by a transfinite induction on the cardinality of $\Gamma$.

In the proof of the lemma, we used the following theorem of P. J. Cohen [1] and Davenport [2].

TheOREM. Let $\Gamma$ be a totally ordered group. Let $E=\left\{\gamma_{1}, \gamma_{2}, \cdots, \gamma_{N}\right\}$ be a fixed set of $N$ positive elements in $\Gamma, N \geqq 3$. Suppose $\mu \in M(G)$, and

$$
\begin{array}{rlrl}
|\mu(\gamma)| \geqq 1 & & \text { for } \gamma \in E, \\
\mu(\gamma) & =0 & & \text { for } \gamma>0, \gamma \notin E .
\end{array}
$$

Then there exists a constant $k$, independent of the group $G$, such that

$$
\|\mu\|>k(\log N / \log \log N)^{1 / 4} \text {. }
$$

This theorem was proven by Cohen [1] and Davenport [2]. An examination of the proofs in these papers shows that they actually obtain the above theorem although they only considered the case where $\mu(\gamma)=0$ for all $\gamma \notin E$. See also Hewitt and Zuckerman [4] for the case in which the torsion subgroup of $\Gamma$ is an arbitrary finite group.

We also have proven

Theorem 2. Let $Q=\left\{\left(n_{1}, \cdots, n_{k}\right) \in Z^{k}: n_{i} \geqq 0\right.$ for $\left.i=1, \cdots, k\right\}$. Suppose $\mu \in M\left(T^{k}\right)$ and $\hat{\mu}(q)$ is an integer for all $q \in Q$. Then there exists $\boldsymbol{a} \lambda \in F\left(T^{k}\right)$ such that $\hat{\mu}(q)=\hat{\lambda}(q)$ for all $q \in Q$. 


\section{BIBLIOGRAPHY}

1. P. J. Cohen, On a conjecture of Littlewood and idempotent measures, Amer. J. Math. 82 (1960), 191-212.

2. H. Davenport, On a theorem of P. J. Cohen, Mathematica 7 (1960), 93-97.

3. H. Helson, On a theorem of Szego, Proc. Amer. Math. Soc. 6 (1955), 235-242.

4. E. Hewitt and H. S. Zuckerman, On a theorem of P. J. Cohen and H. Davenport, Proc. Amer. Math. Soc. 14 (1963), 847-855.

5. T. Ito and I. Amemiya, $A$ simple proof of the theorem of P. J. Cohen, Bull. Amer. Math. Soc. 70 (1964), 774-776.

6. W. Rudin, Permutations of Taylor coefficients of bounded functions, Duke Math. J. 28 (1961), 537-543.

7. - Fourier analysis on groups, Interscience, New York, 1962.

University of Wisconsin and University of Michigan 